\title{
A brief case series of radiation associated myelopathy
}

\author{
Sena D. Bünül, MD, Cansu E. Sarıkaya, MD, Onural Öztürk, MD, Caner Sarıaya, MD.
}

\begin{abstract}

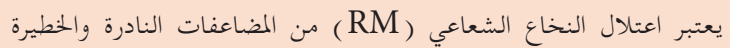

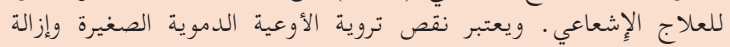

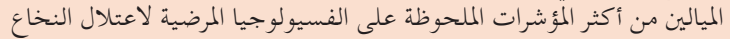

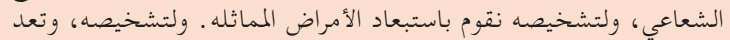

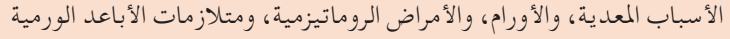

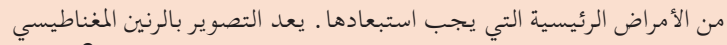

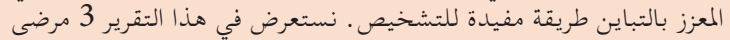

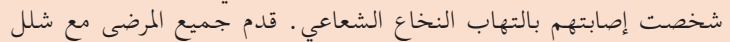

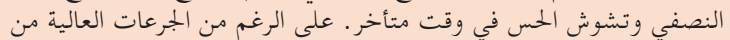

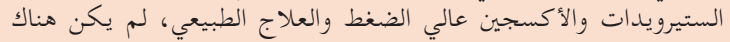
تمسن سريري في المتابعة بعد عام واحد.
\end{abstract}

Radiation myelopathy (RM) is a rare but serious complication of radiotherapy. Small vessel ischemia and demyelination are some of the more remarkable indications of RM's pathophysiology. The RM is a diagnosis of exclusion. In the differential diagnosis, infectious causes, tumors, rheumatic diseases, and paraneoplastic syndromes are the main diseases that should be excluded. Contrast-enhanced magnetic resonance imaging (MRI) is a useful method for diagnosis. We present a report on 3 patients diagnosed with radiation myelitis. All the patients presented with late paraparasia and paresthesia. Despite high-dose steroids, hyperbaric oxygen, and physical therapy, there was no clinical improvement at the follow-up one year later.

Neurosciences 2021; Vol. 26 (4): 392-395 doi: 10.17712/nsj.2021.4.20210071

From the Department of Neurology (Bünül, Sarkaya), Faculty of Medicine, Kocaeli University, Kocaeli, from the Department of Neurosurgery (Sarkkaya), University of Health Sciences Istanbul Umraniye Training and Research Hospital, and from Seka State Hospital (Öztürk), Kocaeli, Turkey.

Received 18th May 2021. Accepted 25th August 2021.

Address correspondence and reprint request to: Dr. Sena D. Bünül, Department of Neurology, Faculty of Medicine, Kocaeli University, Kocaeli, Turkey. E-mail: destansena@gmail.com

ORICID ID: https://orcid.org/ 0000-0003-4999-2787

$\mathrm{R}^{2}$ adiation myelopathy (RM) is defined as a rare but serious complication that occurs as a result of the spinal cord being exposed to ionizing radiation. The spinal cord is the dose-limiting organ in radiotherapy because of its radiation sensitivity and complications that can occur from radiation exposure. Many centers make dose restrictions and regulations for this reason.

The RM is examined under 2 different classifications based on the time of its occurrence. The form that occurs between 6 weeks and 6 months after radiotherapy is called early or transient radiation myelopathy, and the form that occurs later than 6 months is called delayed or progressive RM. ${ }^{1}$ Although the pathophysiology of damage to the central nervous system is unclear, oligodendrocytes and endothelial cells seem to be the main targets. ${ }^{2}$ Although glial cells and demyelination, which are less sensitive and have a shorter latency than endothelial cells, are responsible for the pathophysiology of early RM, endothelial cells and vascular ischemia are responsible for the pathophysiology of late RM. ${ }^{3}$

Clinical findings can vary widely, from minor neurological defects to major motor and sensory deficits. $\mathrm{RM}$ is a diagnosis of exclusion, and MRI with contrast is helpful in making the diagnosis. In the differential diagnosis, infectious causes, tumors, rheumatic diseases, and paraneoplastic syndromes are the main diseases that should be excluded.

Although there are no proven long-term treatments, steroids, hyperbaric oxygen, and physical therapy modalities are among the treatments applied. There are experimental studies on ramipril as a preventative option, and promising results have been reported. ${ }^{4}$ Presenting 3 cases of this rare complication will contribute to the literature.

Case Report. In the current case report, we report 3 cases with similar characteristics. The characteristics of the cases are explained in detail, and the summary information is also presented in Figure 1.

Patient information and clinical findings. Case 1. A 70-year-old male patient was admitted to our neurology

Disclosure. The authors declare no conflicting interests, support or funding from any drug company. 
clinic with paraparesis and paresthesia. From his medical history, it was discovered that he had undergone surgery for chondrosarcoma at the T4-7 vertebral level two years prior and received 54 Gy radiotherapy between T4-7 for one month after the operation. There was no history of motor weakness before radiotherapy, and neurological examination revealed that his bilateral lower extremity motor strength was $5 / 5$ according to the Oxford scale; patellar reflexes were normal, and the Babinski sign was bilaterally negative. No myelitis-related finding was found in the imaging before radiotherapy (Figure 2 A-B). After radiotherapy, neurological examination revealed that bilateral lower extremity motor strength was $3 / 5$ based on the Oxford scale; patellar reflexes were increased, and the Babinski sign was bilaterally positive.

Case 2. A 44-year-old female patient was admitted to our clinic with paraparesis. The patient had surgery for ependymoma at the T7-10 vertebral level 3 years prior. She was operated on for the second time one year later because of recurrence. She received 50.4 Gy radiotherapy for one month after her last operation, and there was no loss of motor strength before radiotherapy. Neurological examination showed that bilateral lower extremity motor strength was $1 / 5$ based on the Oxford scale; patellar reflexes were increased, and the Babinski sign was bilaterally positive.

Case 3. A 47-year-old male patient was admitted to our clinic with paraparesis and paresthesia. From his medical history, we found that the patient had received 52 Gy radiotherapy for one month because of thoracic vertebra metastasis one year earlier. Neurological examination showed that bilateral lower extremity motor strength was $1 / 5$ based on the Oxford scale; patellar reflexes were increased, and the Babinski sign was bilaterally positive.

Diagnostic assessment. In all 3 cases, contrastenhanced MRI was performed for spinal cord imaging. Atrophy was observed only in case 2, while T-2 hyperintense changes were present in all cases. These changes were seen at the T5 vertebral level (Fig $2 \mathrm{C}-\mathrm{D}$ ) in

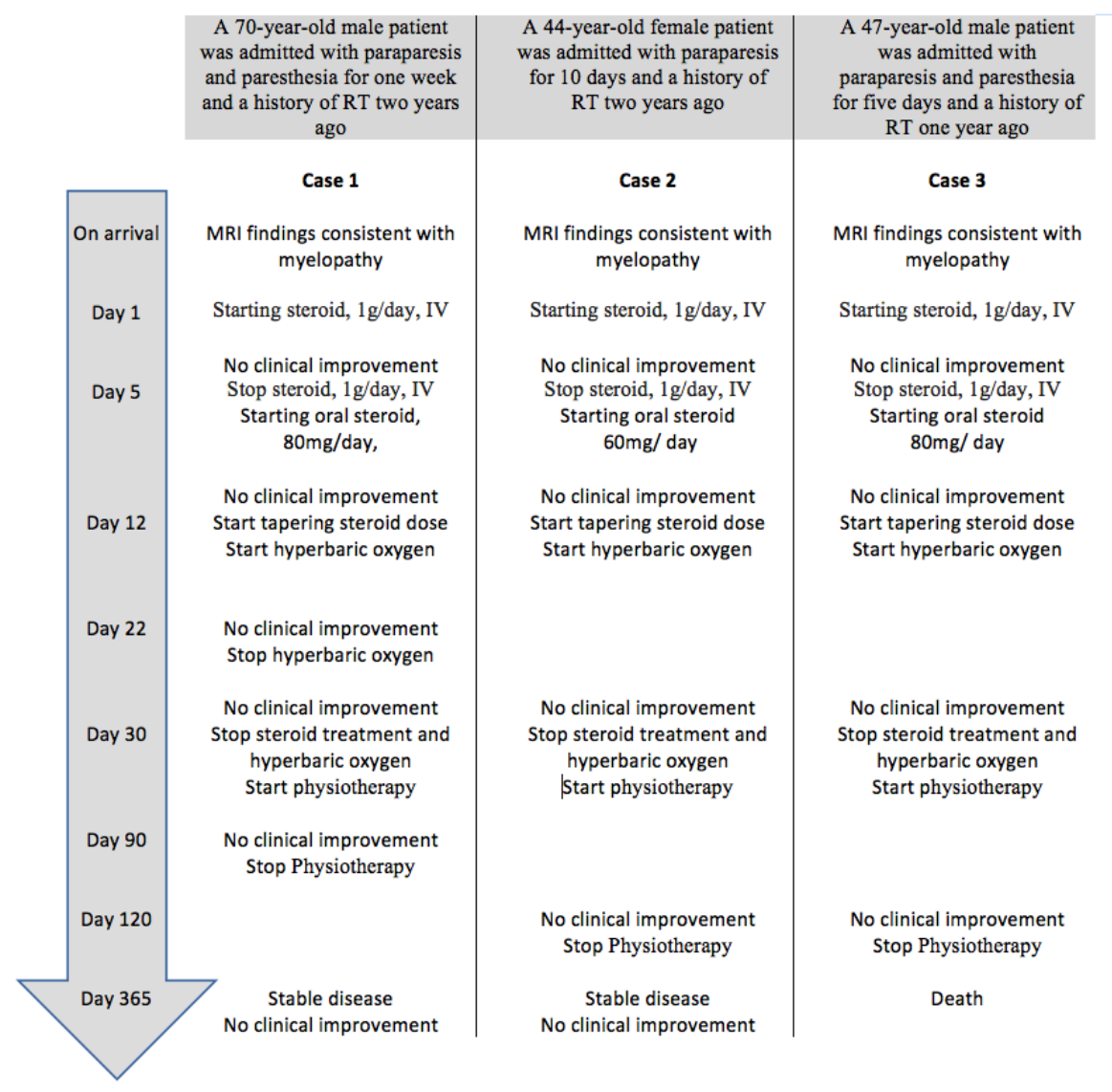

Figure 1 - Timeline of the clinical course of the patients and outcomes. 
Table 1 - Summary of case characteristics.

\begin{tabular}{|c|c|c|c|}
\hline & Case 1 & Case 2 & Case 3 \\
\hline \multicolumn{4}{|l|}{ Patient information } \\
\hline Gender & M & $\mathrm{F}$ & M \\
\hline Age (yr) & 70 & 44 & 47 \\
\hline \multicolumn{4}{|l|}{ Clinical findings } \\
\hline $\begin{array}{l}\text { Time to symptoms onset from } \\
\text { RT (mo) }\end{array}$ & 23 & 23 & 12 \\
\hline RT Dose (Gy) & 54 & 50.4 & 52 \\
\hline
\end{tabular}

MRI Findings

Treatment

Follow up

Duration (mo)

Clinical course
Hyperintensity on a T2weighted sagittal image at the T5 vertebra level

High dose steroid Hyperbaric oxygen Physiotherapy

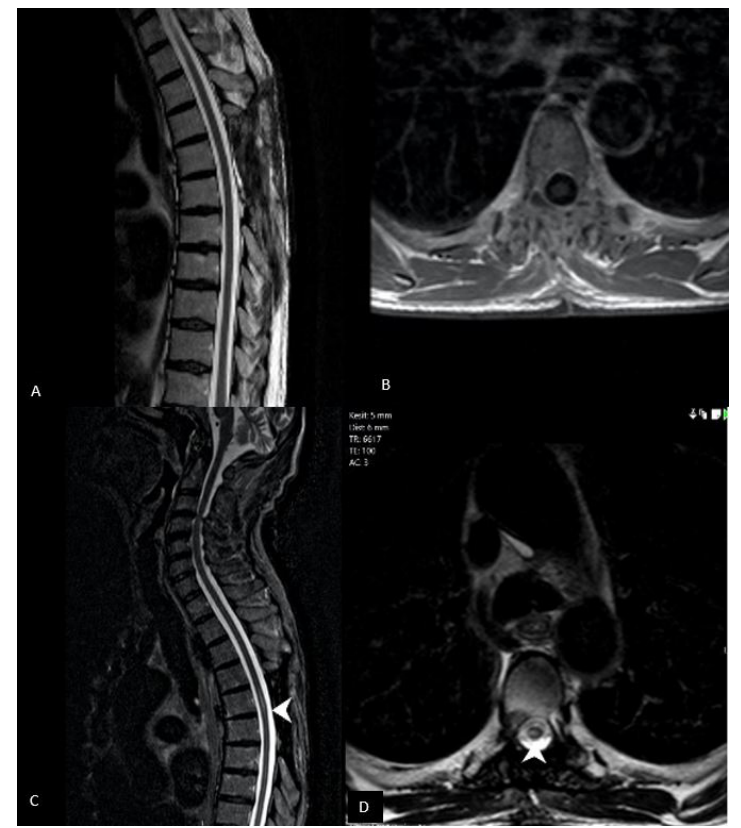

Figure 2 - Magnetic Resonance Imaging A) No hyperintensity on T2weighted sagittal image, B) No hyperintensity on $\mathrm{t} 2$-weighted axial images, C) Central Hyperintensity on a T2-weighted sagittal image at the T5 vertebra level is consistent with myelitis, D) T2-weighted signal increase on axial image at the same level.

case 1 , at the T7-T9 vertebra levels in case 2 (Fig $3 \mathrm{~A}-\mathrm{B}$ ), and at the T9-L1 levels in case 3 (Fig 3 C-D ). These findings are consistent with the regions in which the cases had previously received radiotherapy.

Therapeutic intervention. When the history, physical examination, and imaging findings of the cases
T2 Weighted signal increase along Long-segment hyperintensity on with central cystic necrotic changes a T2-weighted sagittal image at on T7-8-9 vertebral levels

High dose steroid Hyperbaric oxygen Physiotherapy the T9-L1 vertebra levels

High dose steroid Hyperbaric oxygen Physiotherapy
1212

No improvement
Death

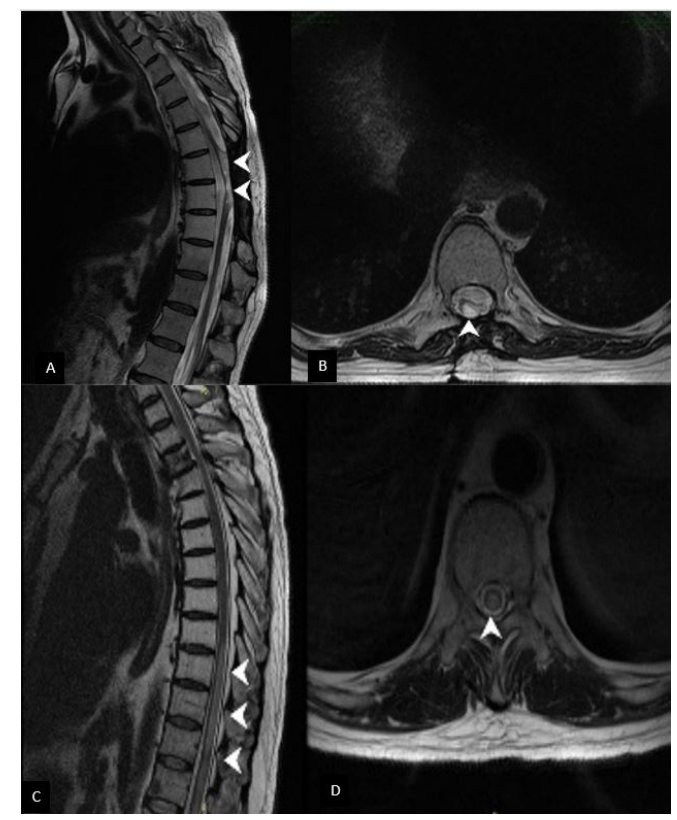

Figure 3 - Magnetic Resonance Imaging A) T2 Weighted signal increase along with central cystic necrotic changes on T7-8-9 vertebral levels. Also note that posterior adhesion of spinal cord is raising suspicion for postoperative arachnoiditis, B) Similar findings on T2-weighted axial image at the T8 vertebra level, C) Long-segment hyperintensity on a T2-weighted sagittal image at the T9-L1 vertebra levels is consistent with myelitis D) Hyperintensity on a T2-weighted axial image at the T10 vertebra level.

were evaluated, RM was considered. Before starting treatment, other diseases that could be included in the differential diagnosis were excluded. All cases received 1 $\mathrm{g} /$ day intravenous steroid therapy for 5 days. Intravenous 
steroid treatment was discontinued, and oral steroid treatment was started after the fifth day in cases that did not show clinical improvement. No improvement was observed in the cases followed up in this way for a week. Hyperbaric oxygen therapy was started for the patients whose steroid therapy was tapered gradually on day 12 . Steroid and hyperbaric oxygen therapy were discontinued in the patients who did not show any improvement on day 30 . The patients were referred to a physical therapy program.

Follow-up and outcome. Despite all the treatments and long-term physiotherapy, there was no improvement in the clinical status of the first 2 cases, and their neurological examinations were similar to the first examinations. The third case died because of the primary disease. All the information on the three cases is summarized in Table 1.

Discussion. The RM is a rare complication. In the study conducted by Khan et al., which examined 20 years of patient cases retrospectively, only 11 cases of RM were identified. 5 Our publication makes an important contribution to the literature by presenting three cases.

The RM manifests within 6 months to 10 years after radiotherapy. Cases of myelopathy developing in the fourth month have also been reported. It has been observed that these patients, especially those with a short latent period, receive repetitive radiation therapy. ${ }^{6}$ At the earliest, late RM presents 6 months after treatment. Our cases also admitted 12-23 months later. In the study conducted by Khan et $\mathrm{al}^{5}$ this period was 17 months (median), which is similar to our cases.

The spinal cord is the dose-limiting organ in radiotherapy because of its radiation sensitivity and complications. Many centers keep the total dose below 45 GY to minimize the risk. It has been reported that the risk of $\mathrm{RM}$ is directly proportional to the amount of spinal cord volume being treated. Gibbs et $\mathrm{al}^{7}$ suggest that caution be used when considering radiosurgery plans that expose more than approximately $1 \mathrm{~cm}^{3}$ of the spinal cord to an 8-Gy or higher dose equivalent. ${ }^{7}$

The radiation tolerance limit of the spinal cord is not clearly known. In retrospective analyses, the risk of RM between 45 and 50 Gy was found to be $0.42 \% .^{8}$ The slightly higher radiation dose in our cases may explain both the occurrence of RM and its irreversible nature.

Normal MRI can be observed at symptom onset. In the following period, hypointensity can be seen in the T1-weighted images, and hyperintensity can be seen in T2-weighted images. Contrast enhancement can be detected in $50 \%$ of cases. ${ }^{\text {? }}$

Primarily, conservative treatments and corticosteroids are used in treating RM. Hyperbaric oxygen therapy is another treatment option although its effectiveness is controversial. ${ }^{10}$

In conclusion, even though RM is not common, it is a serious complication. It is important to limit the amount of spinal cord volume treated and the total radiation dose to prevent the risk of myelopathy.

Acknowledgment. We would like to thank Scribendi Editing for editing and reviewing this manuscript's writing.

\section{References}

1. Schiff D, Arrillaga I, Wen PY. Cancer Neurology in Clinical Practice: Neurological Complications of Cancer and Its Treatment: Springer; 2017.

2. Lu F, Wong C. Radiation-induced apoptosis of oligodendrocytes and its association with increased ceramide and down-regulated protein kinase B/Akt activity. Int J Radiat Biol 2004; 80: 39-51.

3. Coderre JA, Morris GM, Micca PL, Hopewell JW, Verhagen I, Kleiboer BJ, et al. Late effects of radiation on the central nervous system: role of vascular endothelial damage and glial stem cell survival. Radiat Res 2006; 166: 495-503.

4. Saager M, Hahn EW, Peschke P, Brons S, Huber PE, Debus J, et al. Ramipril reduces incidence and prolongates latency time of radiation-induced rat myelopathy after photon and carbon ion irradiation. J Radiat Res 2020; 61: 791-798.

5. Khan M, Ambady P, Kimbrough D, Shoemaker T, Terezakis S, Blakeley J, et al. Radiation-induced myelitis: initial and follow-up MRI and clinical features in patients at a single tertiary care institution during 20 years. AJNR Am J Neuroradiol 2018; 39: 1576-1581.

6. Lewitzki V, Andratschke N, Kuhnt T, Hildebrandt G. Radiation myelitis after hypofractionated radiotherapy with concomitant gefitinib. Radiat Oncol 2015; 10: 29.

7. Gibbs IC, Patil C, Gerszten PC, Adler Jr JR, Burton SA. Delayed radiation-induced myelopathy after spinal radiosurgery. Neurosurgery 2009; 64: A67-A72.

8. Wong C, Fehlings M, Sahgal A. Pathobiology of radiation myelopathy and strategies to mitigate injury. Spinal Cord 2015; 53: 574-580.

9. Wang P, Shen W, Jan J. Serial MRI changes in radiation myelopathy. Neuroradiology 1995; 37: 374-377.

10. Angibaud G, Ducasse J, Baille G, Clanet M. [Potential value of hyperbaric oxygenation in the treatment of post-radiation myelopathies]. Rev Neurol (Paris) 1995; 151: 661-666. French 